ARTIGO ORIGINAL ORIGINAL ARTICLE

\section{Modelo de Cox para análise da sobrevida das empresas de plano de saúde no Brasil}

\author{
Cox model for survival analysis of health \\ insurance companies in Brazil \\ Silvando Carmo de Oliveira', Renan Moritz Varnier Rodrigues de Almeida² \\ DOI: 10.21115/JBES.v11.n1.p57-63
}

\section{Palavras-chave:}

análise de sobrevivência, empresas de plano de saúde, caracterização de dados de sobrevivência, modelo de Cox

\section{Keywords:}

survival analysis, health insurance companies, survival data modeling, survival data characterization, Cox model

\section{RESUMO}

Objetivo: No Brasil, estudos sobre o tempo de vida das operadoras de planos de saúde são escassos. Assim, o artigo tem o objetivo de investigar fatores econômicos que explicam a sobrevida de empresas de plano de saúde. Métodos: Foi utilizada a técnica estatística denominada análise de sobrevivência, por meio do modelo semiparamétrico de Cox. Os dados foram obtidos no site da ANS (agência reguladora do setor) e referem-se a 929 operadoras de todas as regiões do país, em 2011-2018. As seguintes variáveis foram analisadas: Beneficiário (número médio de beneficiário por operadora), Porte (Porte 1: até 20 mil beneficiários, Porte 2: entre 20 mil e 100 mil beneficiários, Porte 3: acima de 100 mil beneficiários), Tempo no Mercado (quantidade de trimestres que a operadora permaneceu no mercado), Receita/Despesa (Muito baixa, Baixa, Alta e Muito alta), Lucro (Operadoras que lucraram e Operadoras que não lucraram), Tipo de Gestão (Gestão sem fins lucrativos e Gestão empresarial) e por último tem-se a variável (dependente) Falência, que indica se a operadora solicitou o encerramento das suas atividades. Resultados: Observou-se uma taxa de mortalidade para operadoras de pequeno porte maior comparativamente às demais, com probabilidade de não sobrevivência no mercado duas vezes menor em relação às empresas de médio porte e três vezes menor se comparada às de grande porte. Conclusões: Empresas de pequeno porte encontram-se em grande desvantagem no panorama brasileiro de operadoras de planos de saúde, qualquer que seja seu tempo de vida no mercado.

Recebido em: 31/01/2019. Aprovado para publicação em: 21/03/2019.

1. Departamento de Economia, Universidade Federal do Ceará (UFC), Campus Sobral, Sobral, CE, Brasil.

2. Departamento de Engenharia Biomédica do Instituto Alberto Luiz Coimbra de Pós-Graduação e Pesquisa de Engenharia (COPPE)/Universidade Federal do Rio de Janeiro (UFRJ), Rio de Janeiro, RJ, Brasil.

Instituição onde o trabalho foi executado: Universidade Federal do Ceará (UFC) - Campus Sobral; Universidade Federal do Rio de Janeiro (UFRJ) - Engenharia Biomédica do Instituto Alberto Luiz Coimbra de Pós-Graduação e Pesquisa de Engenharia (COPPE)/UFRJ.

Fontes de financiamento: $O$ presente estudo foi financiado pelo Conselho Nacional de Desenvolvimento Científico e Tecnológico (CNPq) no Programa Institucional de Pós-Doutorado Júnior (PDJ).

Autor correspondente: Silvando Carmo de Oliveira. Universidade Federal do Ceará - Campus Sobral. Rua Cel. Estanislau Frota, 563, Centro, Sobral, CE, Brasil. CEP: 62010-560. Telefone: +55 (85) 3695-4601. E-mail: scaoli.oliveira@gmail.com 


\section{Introdução}

A recessão econômica que assolou o Brasil nestes últimos quatro anos afetou negativamente o crescimento do número de beneficiários nos planos privados de saúde. Em virtude da tímida recuperação da economia em 2017, parece estar ocorrendo uma reversão da tendência do número de brasileiros com planos de saúde, e esse impacto positivo começa a ser percebido, segundo a Agência Nacional de Saúde Suplementar (ANS), a partir de 2018. No segundo trimestre de 2018, a quantidade de beneficiários de planos de saúde voltou a crescer, apurando 0,3\% em relação ao trimestre anterior e aumento de 132 mil novos beneficiários (Cenário Saúde, 2018).

A atuação dos planos privados de saúde remonta à década de 1950, porém sua regulamentação se deve à Lei Orgânica da Saúde no 8.080/1990, que dispôs sobre o segmento conhecido como "complementar", controlado pelo Sistema Único de Saúde (SUS). Por outro lado, o setor "suplementar", no qual um indivíduo ou empresa faz a opção por planos de saúde privados, teve a sua regulamentação na Lei no 9.656, de junho de 1988, e em janeiro de 2000 criou-se a ANS destinada à regulamentação desse setor (http://www.ans.gov.br/ aans/quem-somos/historico).

Devido à assimetria de informação e ao evento conhecido como seleção adversa, a ANS tem papel crucial na regulamentação dos contratos oferecidos pelos planos privados de saúde, e a entrada e a saída das operadoras no mercado estão fortemente regulamentadas, ou seja, devem seguir critérios estritamente definidos em lei. Estamos assumindo que a assimetria de informações acontece quando as operadoras e também os beneficiários do plano não possuem todas as informações necessárias sobre os bens e serviços que estão sendo ofertados e/ou adquiridos, ou seja, estamos admitindo a não existência de informação perfeita das partes envolvidas. Como consequência da assimetria de informação, a seleção adversa ocorre quando o beneficiário faz uso do plano com probabilidade acima da média ou entra com uma doença preexistente; do lado da operadora se dá quando passa a cobrar preços diferenciados por faixa etária ou sexo, penalizando, assim, os beneficiários que fazem uso do plano dentro da média de mercado.

Por exemplo, o aporte mínimo financeiro deve ser incorporado ao patrimônio líquido da empresa em até seis anos, quando então a empresa sai da condição de "registro provisório" para "permanente"; e a saída do mercado é condicionada ao cumprimento de regulamentações relativas à continuidade do acesso aos serviços prestados. Assim, para o encerramento das atividades, uma operadora deve garantir acesso continuado aos serviços oferecidos, por meio da transferência de carteira ou opção de migração para outro plano, sendo mantidos os prazos de carência já cumpridos.
Também não deve haver nova taxa de adesão, pré-mensalidade ou outras taxas relativas ao novo plano.

Nesse contexto, este artigo tem o objetivo de analisar a sobrevida das empresas brasileiras de planos de saúde durante o período de 2011 a 2018. Para tanto, lança mão de técnicas estatísticas de modelagem não lineares de sobrevivência cujo estimador não paramétrico para a função sobrevivência é resultante de um modelo Cox.

\section{Métodos}

\section{Análise de sobrevivência}

O termo "análise de sobrevivência" está muitas vezes ligado a situações médicas envolvendo dados censurados. No entanto, essa técnica é perfeitamente apropriada às áreas da engenharia, ciências sociais e economia. Nesta última área, por exemplo, pesquisadores trabalham em estudos de mudanças de empregos, desempregos, promoções e aposentadoria (Colosimo \& Giolo, 2006).

O principal interesse da análise de sobrevivência é especificar a variável aleatória não negativa "tempo de falha" (tempo até a ocorrência de um evento de interesse). Isso é feito por meio de uma função de sobrevivência ou pela função de taxa de falha (Colosimo \& Giolo, 2006; Bastos, 2006). A função de sobrevivência é definida como a probabilidade de uma observação não falhar até um certo tempo $t$ (Colosimo \& Giolo, 2006):

$S(t)=P(T \leq t)$

em que: T: tempo de falha; $t$ : tempo de sobrevida.

Há técnicas paramétricas e não paramétricas para estimar essa função de sobrevivência, sendo muito utilizado o estimador apresentado por (Kaplan \& Meier, 1958):

$$
\hat{S}(t) \prod_{j: \mathrm{j}, \mathrm{t} t}\left(1-\frac{d_{j}}{n_{j}}\right)
$$

em que $t_{1}<t_{2} \ldots<t_{k^{\prime}}$, os $k$ tempos distintos e ordenados de falha, $d_{j}$ o número de falhas em $t_{j} j=1, \ldots, k$, e $n_{j}$, e $n_{j}$ é o número de indivíduos sob risco em $t_{i}$ ou seja, os indivíduos que não falharam e não foram censurados até o instante imediatamente anterior a $t_{i}$

\section{Modelo de regressão de Cox}

A introdução desse modelo permite analisar dados decorrentes de estudo de tempo até a ocorrência de um evento de interesse, sendo ele ajustado por covariáveis. O modelo de Cox para uma única covariável pode ser descrito como:

$$
\begin{aligned}
& \lambda_{1}(t)=\lambda_{0}(t) \exp \{\beta x\} \text {, ou seja, } \lambda_{1}(t)=\lambda_{0}(t) \exp \{\beta\} \text {, sex }=\lambda_{0}(t), \\
& \text { sex }=0
\end{aligned}
$$

Sendo $\lambda_{1}(t)$ a taxa de falha para um dado i qualquer.

Agora, considere-se p covariáveis, de modo que $x$ seja um vetor com os seguintes componentes $x=\left(x_{1}, \ldots, x_{p}\right)^{\top}$. Então a expressão geral do modelo Cox fica: 
$\lambda_{1}(t)=\lambda_{0}(t) g\left\{x^{\top} \beta\right\}$.

Sendo $g$ uma função não negativa e assuma que $g(0)=1$.

Logo, esse modelo é composto pelo produto de dois componentes, um não paramétrico e outro paramétrico. O componente não paramétrico $\Lambda_{0}(t)$ não é especificado e é uma função não negativa no tempo. Ele é usualmente chamado de função de base ou basal, pois $\lambda(t)=\lambda_{0}(t)$ quando $x=0$. $O$ componente paramétrico é frequentemente usado na seguinte forma multiplicativa:

$g\left\{x^{\top} \beta\right\}=\exp \left\{\beta_{1} x_{1}+\ldots+\beta_{p} x_{p}\right\}$, em que $\beta$ é o vetor de parâmetros associados às covariáveis. Essa forma garante que $\lambda(t)$ seja sempre não negativa.

Esse modelo é também denominado de modelo de riscos proporcionais, pois a razão das taxas de falha de dois indivíduos i e j é constante no tempo e é dada por:

$$
\frac{\lambda_{i}(t)}{\lambda_{j}(t)}=\frac{\lambda_{o}(t) \exp \left\{x_{i}^{\top} \beta\right\}}{\lambda_{0}(t) \exp \left\{x_{j}^{\top} \beta\right\}}=\exp \left\{x_{i}^{\top} \beta-x_{j}^{\top} \beta\right\}
$$

\section{Caracterização de operadoras em saúde no país}

Segundo a definição que consta no artigo $4^{\circ}$ da Resolução Normativa no 392/2015', que trata de ativos garantidores, o tamanho das operadoras, segundo o porte, tem como referência o Sistema de Informações de Beneficiários (SIB), conforme os critérios: a) operadora de pequeno porte: número de beneficiários inferior a 20 mil; b) operadora de médio porte: número de beneficiários entre 20 mil e 100 mil; e c) operadora de grande porte: número de beneficiários superior a 100 mil. Neste trabalho será usado como Proxy [diferentemente da definição do Serviço Brasileiro de Apoio às Micro e Pequenas Empresas (Sebrae), que estabelece critérios de faturamento] o tamanho da carteira das operadoras.

\section{Obtenção de dados e seleção de covariáveis}

A Tabela 1 indica as variáveis utilizadas neste estudo. As variáveis foram obtidas no site da ANS (http://www.ans.gov. br/), em seu sistema Tabnet. Ao todo, coletaram-se dados de 929 operadoras de planos de saúde especializadas apenas na área de medicina, ou seja, operadoras cujas modalidades são referentes a planos dentários não foram consideradas. No estudo, "beneficiários" significam a média de beneficiários no período estudado e o porte de cada operadora foi definido de acordo com o número médio de beneficiários. Assim, operadoras em que a média de beneficiários não ultrapassou 20 mil foram consideradas de Porte 1, já as que têm mais de

Agência Nacional de Saúde, Resolução Normativa - RN n 392, de 9 de dezembro de 2015. Pesquisa feita no site http://www.ans.gov.br/ component/legislacao/?view=legislacao\&task=TextoLei\&format=raw\&i $\mathrm{d}=\mathrm{MzE} 1 \mathrm{Mg}==\mathrm{em}$ 01/01/2019.
Tabela 1. Descrição das variáveis estudadas - 929 operadoras de saúde, Brasil, 2011-2018

\begin{tabular}{ll}
\hline Variável & Descrição \\
\hline Operadora & Empresa que fornece o plano de saúde \\
\hline Beneficiário & $\begin{array}{l}\text { Número médio de beneficiários } \\
\text { durante o tempo (em trimestres) } \\
\text { da operadora no mercado }\end{array}$ \\
\hline Porte & $\begin{array}{l}\text { Porte da operadora (1 = até } 20 \text { mil } \\
\text { beneficiários, } 2=\text { entre } 20 \text { mil e } \\
100 \text { mil beneficiários, } 3=\text { acima } \\
\text { de 100 mil beneficiários })\end{array}$ \\
\hline Tempo no mercado & $\begin{array}{l}\text { Número de trimestres que a operadora } \\
\text { permaneceu no mercado }\end{array}$ \\
\hline Receita & $\begin{array}{l}\text { Quantidade total de receitas } \\
\text { da operadora }\end{array}$ \\
\hline Despesa & $\begin{array}{l}\text { Quantidade total de despesas } \\
\text { da operadora }\end{array}$ \\
\hline Lucro & $\begin{array}{l}\text { (0 = operadoras que não lucraram, } \\
1=\text { operadoras que lucraram) }\end{array}$ \\
\hline Tipo de gestão & $\begin{array}{l}\text { (0 = sem fins lucrativos, 1 = empresarial) } \\
\text { Faliúvel que indica se a operadora } \\
\text { faliu (0 = não, 1 = sim) }\end{array}$ \\
\hline
\end{tabular}

Fonte: O Autor.

20 mil e menos de 100 mil foram consideradas de Porte 2 e as que têm acima de 100 mil foram consideradas de Porte 3 . A variável Porte é construída a partir da definição do número de beneficiários de acordo com os critérios estabelecidos pela ANS.

Os dados relativos à despesa representam o resultado da soma de despesas assistencial, administrativa, de comercialização e outras despesas operacionais, e a receita foi calculada como a soma das receitas de contraprestações e outras receitas operacionais. Essa variável foi dividida em quatro classes, denominadas de: Muito baixa $\left(5,9 \times 10^{7}\right)$, Baixa (entre $6,0 \times 10^{7}$ e 2,3 $\times 10^{8}$ ), Alta (entre $2,4 \times 10^{8}$ e 6,9 $\times 10^{8}$ ) e Muito alta (acima de $7 \times 10^{8}$ ). A variável Lucro foi então definida como binária: 1 para empresas com receitas maiores que despesas e 0 caso contrário.

Foram considerados dois tipos de gestões. O primeiro tipo leva em conta apenas as operadoras sem fins lucrativos, cujas modalidades são: Autogestão, Cooperativa Médica e Filantropia. O outro tipo foi denominado de Empresarial, fazendo parte desse grupo das modalidades: Medicina de Grupo e Seguradora Especializada em Saúde.

\section{Análise descritiva}

As variáveis foram inicialmente caracterizadas por meio de suas médias (variáveis contínuas) e percentuais (categóricas) e, a seguir, foram estimadas curvas de Kaplan-Meier para as 
seguintes variáveis: Porte da operadora, Tipo de gestão, Tempo de operação e Lucro. A hipótese nula de inexistência de diferença ente curvas relativas a categorias de uma variável foi testada pelo valor-p em um teste Log-Rank (Mantel, 1966).

\section{Modelagem}

No modelo, o período de observação inicia-se no ano de jun./2011 e finda em jun./2018, e o tempo de duração das operadoras no mercado é dado em trimestres. $O$ tempo de duração das operadoras no mercado foi a variável dependente ou resposta. Dessa forma, foi contabilizado o número de trimestres em que a operadora apresentou pelo menos um beneficiário a partir de jun./2011 até jun./2018. Algumas empresas iniciaram suas atividades após o primeiro trimestre do estudo, portanto a análise avalia o tempo de sobrevivência a partir do começo do estudo ou do início de suas atividades. Foi considerado como "censura" qualquer operadora que não faliu durante o período estudado, independentemente do seu início de atividades. Nesse contexto, adotou-se "censura à direita do tipo I" (o estudo será terminado após um período preestabelecido de tempo e a falência das operadoras ainda não ocorreu até o final do estudo).

Para selecionar as covariáveis do modelo de Cox, são utilizados sete passos, e em cada um deles são feitos testes de hipóteses com o intuito de verificar a importância das variáveis no modelo. Nesse contexto, será utilizado o Teste da Razão de Verossimilhanças Generalizadas (TRVG), e sob hipótese nula ele segue uma distribuição qui-quadrado.

Apresentado por Wilks (1938), o teste tem como interesse obter o máximo da log-verossimilhança para os modelos com todos os parâmetros fixos e modelos com um número reduzido de parâmetros. E para obter a estatística de teste, utilizamos duas vezes a diferença log-verossimilhança sob esses dois modelos.

O teste da Razão de Verossimilhanças (RV) pode ser útil quando temos o interesse de verificar se o modelo com mais parâmetros não se ajusta significativamente melhor do que um modelo restrito (com um número menor de parâmetros). Além disso, o teste da Razão de Verossimilhanças, em particular, não é indicado quando o tamanho amostral é pequeno, tornando, assim, os procedimentos inferenciais aproximados pouco satisfatórios (Melo et al., 2009; Stein, 2008). A estatística da RV é dada por:

$$
Q_{r v}=2\left(L_{1}-L_{2}\right),
$$

em que $L$, representa o máximo da log-verossimilhança sob o modelo restrito (encaixado) e $L_{2}$ é a log-verossimilhança correspondente do modelo com $r$ variáveis adicionais e 1(.) corresponde à log-verossimilhança. Temos que $Q_{r v}$ segue uma distribuição qui-quadrado com $r$ graus de liberdade.

Em todos os testes, utilizou-se $a=0,05$. Para o modelo final, foram calculados também os intervalos de confiança de 95\% para as variáveis remanescentes.
Para o ajuste computacional do modelo, foi utilizado o pacote survival, disponível para o software R em https:// cran.r-project.org/web/packages/survival/index.html (R Core Team, 2018).

\section{Resultados}

A Tabela 2 ilustra o conjunto de dados das 929 operadoras analisadas. "Operadoras falidas" foram aquelas que não sobreviveram até jun./2018. A análise de Kaplan-Meyer (Figura 1) indica que as operadoras de menor porte têm a menor probabilidade de sobrevivência (Portes 2 e 3 possuem curvas de sobrevivência semelhantes). Relativamente aos tipos de gestão, há diferença significativa entre as duas curvas de sobrevivência, e, quanto menor a receita da empresa, menor a probabilidade de sobrevivência. Como esperado, operadoras com lucro tiveram maior probabilidade de sobrevivência (Tabela 3).

A partir dos procedimentos descritos anteriormente, obteve-se como modelo final de Cox a equação:

$$
\lambda(t)=\lambda_{0}(t) \exp \left\{\beta_{1} X_{1}+\beta_{2} X_{2}+\beta_{3} X_{3}+\beta_{4} X_{4}\right\}
$$

em que: $X_{i}$ : Porte $2 ; X_{2}$ : Porte $3 ; X_{3}$ : Gestão empresarial; $X_{4}$ : Lucro.

Os coeficientes desse modelo (com as seguintes categorias de referência: Porte 1, Gestão sem fins lucrativos e Operadoras que não lucraram) são apresentados na Tabela 4. Não foram detectadas interações entre as variáveis do modelo, e a suposição de riscos proporcionais pode ser aceita para to-

Tabela 2. Conjunto de dados 929 operadoras de saúde, Brasil, 2011-2018

\begin{tabular}{lcc}
\hline Porte & Percentual & $\begin{array}{c}\text { Percentual de } \\
\text { Sobrevivência }\end{array}$ \\
\hline Tipo & $61,57 \%$ & $71,15 \%$ \\
\hline Até 20 mil beneficiários & $29,81 \%$ & $86,28 \%$ \\
\hline Entre 20 mil e 100 mil beneficiários & $8,61 \%$ & $87,50 \%$ \\
\hline Acima de 100 mil beneficiários & & \\
\hline Lucro & Percentual & $\begin{array}{c}\text { Percentual de } \\
\text { Sobrevivência }\end{array}$ \\
\hline Tipo & $33,80 \%$ & $59,23 \%$ \\
\hline Não & $66,20 \%$ & $86,17 \%$ \\
\hline Sim & & \\
\hline Gestão & Percentual & $\begin{array}{c}\text { Percentual de } \\
\text { Sobrevivência }\end{array}$ \\
\hline Tipo & $58,56 \%$ & $83,63 \%$ \\
\hline Sem fins lucrativos & $41,44 \%$ & $67,79 \%$ \\
\hline Empresarial & & \\
\hline
\end{tabular}

Fonte: O Autor. 

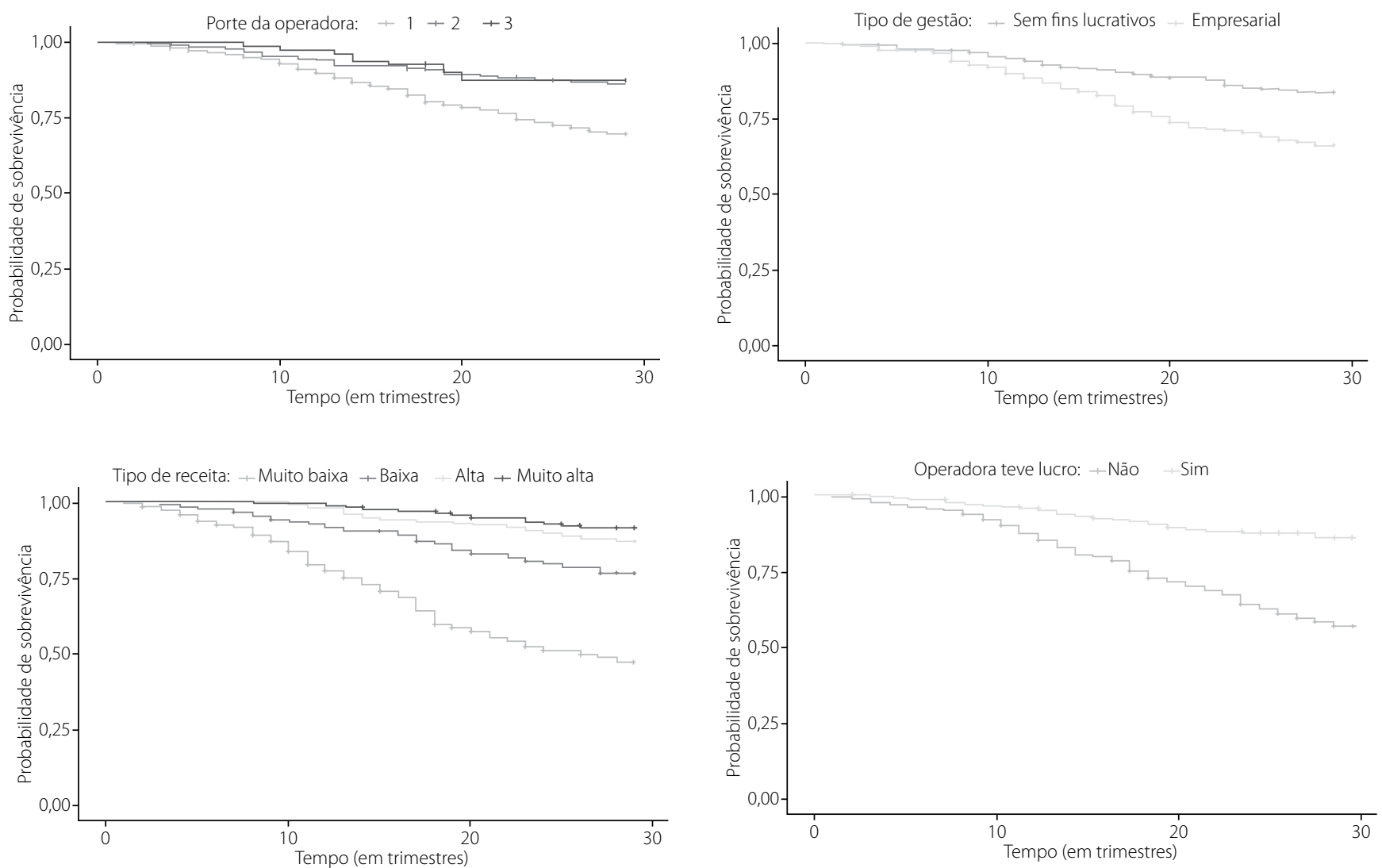

Fonte: O Autor.

Figura 1. Curvas de sobrevivência estimadas pelo método de Kaplan-Meier - 929 operadoras de saúde, Brasil, $2011-2018$.

Tabela 3. Estatísticas de comparação das curvas de sobrevivência dos Tipo de gestão, Lucro, Receita, Despesa e Porte das operadoras estudadas - 929 operadoras de saúde, Brasil, 2011-2018

Teste (Valor-p)

\begin{tabular}{|c|c|c|c|}
\hline \multirow[b]{2}{*}{ Variável } & & \multirow[b]{2}{*}{ Log-Rank } & \multirow[b]{2}{*}{ Wilcoxon Generalizado } \\
\hline & & & \\
\hline \multicolumn{4}{|c|}{ Tipo de Gestão } \\
\hline & Sem fins lucrativos $x$ Empresarial & $<0,0001$ & $<0,0001$ \\
\hline \multicolumn{4}{|l|}{ Lucro } \\
\hline & Empresa lucrou x Empresa não lucrou & $<0,0001$ & $<0,0001$ \\
\hline \multicolumn{4}{|c|}{ Receita/Despesa } \\
\hline & Muito baixa x Baixa & $<0,0001$ & $<0,0001$ \\
\hline & Muito baixa x Alta & $<0,0001$ & $<0,0001$ \\
\hline & Muito baixa x Muito alta & $<0,0001$ & $<0,0001$ \\
\hline & Baixa x Alta & 0,0026 & 0,0017 \\
\hline & Baixa x Muito alta & $<0,0001$ & $<0,0001$ \\
\hline & Alta x Muito alta & 0,1245 & 0,124 \\
\hline \multicolumn{4}{|l|}{ Porte } \\
\hline & Baixo x Médio & $<0,0001$ & $<0,0001$ \\
\hline & Baixo x Alto & 0,0026 & 0,7624 \\
\hline & Médio x Alto & 0,0012 & 0,7438 \\
\hline
\end{tabular}

Fonte: O Autor. 
Tabela 4. Resultado do ajuste do modelo de regressão de Cox

\begin{tabular}{lccc}
\hline Covariável (Categorias) & Estimativa & Erro-padrão & Valor-p \\
\hline Porte 2 & $-0,706$ & 0,181 & $<0,0001$ \\
\hline Porte 3 & $-0,847$ & 0,326 & 0,010 \\
\hline Empresarial & 0,710 & 0,140 & $<0,0001$ \\
\hline Lucro & $-1,144$ & 0,141 & $<0,0001$ \\
\hline
\end{tabular}

Fonte: O Autor.

Tabela 5. Testes da proporcionalidade das taxas de falhas no modelo de Cox

\begin{tabular}{lccc}
\hline Covariáveis (categorias) & $\boldsymbol{\rho}$ & $\mathbf{x}^{\mathbf{2}}$ & Valor- $\mathbf{p}$ \\
\hline Porte 2 & $-0,102$ & 2,250 & 0,134 \\
\hline Porte 3 & $-0,024$ & 0,122 & 0,727 \\
\hline Empresarial & 0,028 & 0,164 & 0,685 \\
\hline Lucro & $-0,125$ & 3,404 & 0,065 \\
\hline Global & - & 6,555 & 0,161 \\
\hline
\end{tabular}

Fonte: O Autor.

das as variáveis. Em suma, pode-se afirmar que: 1. O risco de falência de operadoras do Porte 1 é duas vezes maior do que o de operadoras do Porte $2(\exp \{0,706\}=2,026) ; 2$. O risco de falência de operadoras do Porte 1 é 2,3 vezes maior do que o de operadoras do Porte $3(\exp \{0,847\}=2,333) ; 3$. As operadoras com o tipo de gestão empresarial têm o risco de falência quase duas vezes maior que empresas sem fins lucrativos; 4. Operadoras que não tiveram lucro possuem risco de falência três vezes maior do que aquelas que lucraram $(\exp \{1,144\}=$ 3,140). Por meio da Tabela 5, observa-se que em nenhuma covariável a suposição básica de riscos proporcionais é rejeitada. Para um bom ajuste, deve-se considerar também a correlação dos riscos com o tempo (p). Ou seja, p próximo de zero indica que não existe tendência entre o risco de falência e o tempo. Por exemplo, se uma operadora tiver risco de falência duas vezes maior que outra durante certo trimestre, esse comportamento deve perdurar durante todo o estudo. Portanto, como a suposição básica de riscos proporcionais não foi rejeitada, considera-se que foi obtido bom ajuste. Para mais detalhes sobre o teste de riscos proporcionais $\left(H_{0}: p=0\right)$, ver Colosimo \& Giolo (2006).

\section{Discussão}

Este estudo investigou a sobrevida das empresas brasileiras de planos de saúde durante o período de 2011 a 2018, com atenção particular às pequenas e médias empresas desse setor. Os resultados principais da pesquisa indicam que as operadoras de planos de saúde de pequeno porte têm probabilidade de não sobrevivência no mercado duas vezes menor em relação às empresas de médio porte e três vezes menor se comparadas às de grande porte, e vale também ressaltar que esse risco de falência independe do tempo que a empresa de pequeno porte atua no mercado, resultado esse que pode ser observado nos resultados do modelo de Cox, que apontou não significativa para a variável tempo de operação.

Como mencionado, existem barreiras à entrada e à saída de novas operadoras no mercado. Assim, a partir de seus marcos regulatórios, as empresas foram obrigadas a se adaptar às novas exigências, aumentando a concentração no mercado das operadoras de médio e grande porte e, consequentemente, com redução ou mortalidade das empresas de pequeno porte. Segundo Corrêa (2016), o número de operadoras entre 2006 e 2015 reduziu-se em cerca de um terço. Uma parte da explicação desse fenômeno deve-se ao aumento dos custos: enquanto as receitas operacionais entre 2008 e 2018 cresceram em média 197\%, as despesas assistenciais, no mesmo período, aumentaram em 212\% (ANS, 2018).

O modelo de Cox indicou que as micro e pequenas empresas (MPEs) e, em especial, as de empresas pequeno porte apresentam risco de falência relativa considerável. No entanto, as MPEs são protagonistas de grande parte dos importantes índices da economia brasileira. Dados do Anuário do Trabalho na Micro e Pequena Empresa (Dieese, 2014), organizado pelo Sebrae (Sebrae/Dieese, 2013), mostraram que em 2013 as MPEs responderam, em média, por 99\% dos estabelecimentos, 52\% dos empregos formais de estabelecimentos privados não agrícolas do país e de quase $42 \%$ da massa de salários paga aos trabalhadores desses estabelecimentos; e setorialmente o comércio manteve-se como a atividade com o maior número de MPEs. Da mesma forma, as pequenas empresas de plano de saúde ativas em outubro de 2018 representam 63\% do total das operadoras; considerando as MPEs, esse percentual sobe para $90 \%$. A maioria dessas MPEs está localizada no interior do país e é responsável pelo atendimento a mais de 6 milhões de beneficiários de planos de saúde (cerca de $10 \%$ do total).

Essas baixas taxas de sobrevivência das MPEs, observadas no presente estudo, sempre têm despertado a atenção dos estudiosos sobre o tema, e Davis (1939) já as estudava no final da década de 1930. Sua pesquisa, realizada com dados da empresa Dun \& Bradstreet, mostra que, em algumas cidades dos Estados Unidos, a mortalidade das MPEs chegava a 77,6\% ao final do terceiro ano de atividade. Em termos gerais, os principais fatores citados pelo autor como responsáveis por essa alta taxa de mortalidade consistiam da falta de mão de obra especializada, falta de infraestrutura, instabilidade política e econômica, e rápida mudança de demanda por parte dos clientes (Filardi, 2006). No Brasil, embora não existam trabalhos específicos na área de saúde, o Sebrae (2016), tomando como base os dados disponibilizados pela Secretaria da Receita Federal entre os anos de 2008 a 2012 (até quatro anos de atividade), identificou os fatores condicionantes do fracasso e do sucesso das MPEs. 


\section{Conclusão}

O principal resultado do trabalho mostra que as pequenas empresas de planos de saúde estão em posição muito desvantajosa no país. Um fator determinante dessa desvantagem é o excesso de regulamentação exigida, o que é reconhecido pela própria ANS, a qual implementou um conjunto de medidas com o objetivo específico de diminuir o excesso de regulamentação (Resolução Normativa no 274, de 20 de outubro de 2011). No entanto, o resultado principal deste trabalho reforça a tese de que tais medidas não surtiram o efeito desejado nas empresas de pequeno porte no setor de saúde no período analisado.

\section{Referências bibliográficas}

Agência Nacional de Saúde Suplementar (ANS). Dados gerais. Available at: http://www.ans.gov.br/perfil-do-setor/dados-gerais. Accessed on: 12/13/2018

Bastos J, Rocha C. Análise de sobrevivência: Conceitos Básicos. Arq Med. 2006;20(5-6):185-7. Available at: http://www.scielo.mec.pt/pdf/am/ v20n5-6/v20n5-6a07.pdf. Accessed on: 10/20/2018.

BNDES - Banco Nacional de Desenvolvimento Econômico e Social. Análise da sobrevivência das firmas brasileiras. Rio de Janeiro: BNDES; 2002. (Série Informe-se, n. 46). Available at: https://goo.gl/kMkyod. Accessed on: 04/09/2019.

Cenário Saúde: Sistema Abramge - Associação Brasileira de Planos de Saúde, Sinamge - Sindicato Nacional das Empresas de Medicina de Grupo, e Sinog - Sindicato Nacional das Empresas de Odontologia de Grupo, vol. 3, no. 2 de 2018.

Colosimo EA, Giolo AR. Análise de sobrevivência aplicada. São Paulo: Blucher; 2006.

Corrêa RA. Análise de aspectos que influenciam o desempenho de operadoras de planos de saúde privados. Universidade FUMEC, Faculdade de Ciências Empresariais - FACE, Curso de Mestrado em Administração. Dissertação de Mestrado em Administração. Belo Horizonte/MG, 2016.

Cox DR. Partial likelihood. Biometrica. 1975;62:269-76.

Cox DR. Regression Models and Life Tables (with discussion). J R Stat Soc Series B Stat Methodol. 1972;34:187-202.

Ercolin CA. Fatores Financeiros determinantes da mortalidade de micro e pequenas empresas [dissertação]. São Paulo: Faculdade de Economia, Administração e Contabilidade da Universidade de São Paulo; 2007.

Filardi LF. Estudo dos fatores contribuintes para a mortalidade precoce de micro e pequenas empresas de São Paulo [tese]. São Paulo: Faculdade de Economia, Administração e Contabilidade da Universidade de São Paulo; 2006.
Fox J, Sá Carvalho M. The RcmdrPlugin.survival Package: Extending the R Commander Interface to Survival Analysis. J Stat Softw. 2012;49(7):1-32.

Hermeto R, Kridi D, Rocha A, Gomes D. A distributed algorithm for semantic collector selection in wireless sensors networks. Journal of Applied Computing Research. 2013;3(1):1-10.

IESS - Instituto de Estudos de Saúde Suplementar. Textos para Discussão nº 67. Regulação da saúde suplementar: teoria e experiências nos países; 2018.

Kaplan EL, Meier P. Nonparametric estimation from incomplete observations. JASA. 1958;53(282):457-81.

Klein JP, Moeschberger ML. Survival Analysis Techniques for Censored and Truncated Data. New York: Springer; 1997.

Lee ET, Go OT. Survival analysis in public health research. Ann Rev Public Health. 1997;18:105-34.

Mantel N. Evaluation of survival data and two new rank order statistics arising in its consideration. Cancer Chemother Rep. 1966;50:163-70.

Melo TFN, Ferrari SLP, Cribari-Neto F. Improved testing inference in mixed linear models. London: Elsevier; 2009.

R Core Team. R: A language and environment for statistical computing. $R$ Foundation for Statistical Computing, Vienna, Austria. 2018. Available at: https://www.R-project.org. Accessed on: 01/13/2009.

Rocha A, Pirmez L, Delicato FC, Lemos E, Santos I, Gomes D, et al. WSNs clustering based on semantic neighborhood relationships. Computer Networks. 2012;56(5):1627-45.

Rocha C, Papoila AL. Análise de Sobrevivência. XVII Congresso da Sociedade Portuguesa de Estatística (SPE); 2009.

Sebrae - Serviço Brasileiro de Apoio às Micro e Pequenas Empresas; Dieese - Departamento Intersindical de Estatística e Estudos Socioeconômicos. Anuário do Trabalho na Micro e Pequena Empresa 2012. Brasília: Sebrae; Dieese; 2012. Available at: https://goo.gl/kWhBvX. Accessed on: 04/09/2019.

Sebrae - Serviço Brasileiro de Apoio às Micro e Pequenas Empresas. Sobrevivência das Empresas no Brasil: Coleção Estudos e Pesquisas. Brasília; 2013. Available at: https://goo.gl/zNi4c1. Accessed on: 04/09/2019.

Sebrae - Serviço Brasileiro de Apoio às Micro e Pequenas Empresas. Sobrevivência das Empresas no Brasil: Coleção estudos e pesquisas. 2016. Disponível em https://goo.gl/zNoxiP. Accessed on: 04/09/2019.

Sebrae/Dieese - Serviço Brasileiro de Apoio às Micro e Pequenas Empresas/ Departamento Intersindical de Estatística e Estudos Socioeconômicos. Anuário do Trabalho na Micro e Pequena Empresa: 2015. São Paulo; 2015. Available at: https://goo.gl/z8Tdkq. Accessed on: 04/09/2019.

Woodall C, Grambsch P, Thomas W. Applying survival analysis to a largescale forest inventory for assessment of tree mortality in Minnesota. Ecological Modelling. 2005;189(1-2):199-208. 